\title{
Tier-Scalable Reconnaissance Missions For The Autonomous Exploration Of Planetary Bodies
}

\author{
Wolfgang Fink ${ }^{(1)}$, James M. Dohm ${ }^{(2,3)}$, Mark A. Tarbell ${ }^{(1)}$, Trent M. Hare ${ }^{(4)}$, Victor R. Baker ${ }^{(2,3)}$, \\ Dirk Schulze-Makuch ${ }^{(5)}$, Roberto Furfaro ${ }^{(6)}$, Alberto G. Fairén ${ }^{(7)}$, Ty P.A. Ferré( ${ }^{(2)}$, \\ Hideaki Miyamoto $^{(8)}$, Goro Komatsu ${ }^{(9)}$, William C. Mahaney ${ }^{(10)}$ \\ ${ }^{(1)}$ California Institute of Technology, Visual and Autonomous Exploration Systems Research Laboratory, Division of \\ Physics, Mathematics and Astronomy, Mail Code 103-33, Pasadena, CA 91125, USA, \\ Email:wfink@autonomy.caltech.edu,mark@autonomy.caltech.edu, Website: http://autonomy.caltech.edu \\ ${ }^{(2)}$ Dept. of Hydrology and Water Resources, University of Arizona, Tucson, AZ, USA, \\ Email: jmd@hwr.arizona.edu, baker@hwr.arizona.edu, ty@hwr.arizona.edu \\ ${ }^{(3)}$ Lunar and Planetary Laboratory, University of Arizona, Tucson, AZ, USA \\ ${ }^{(4)}$ United States Geologic Survey, Flagstaff, AZ, USA, \\ Email: thare@usgs.gov \\ ${ }^{(5)}$ Dept. of Geology, Washington State University, Pullman, WA, USA, \\ Email: dirksm@wsu.edu \\ ${ }^{(6)}$ Aerospace and Mechanical Engineering Dept., University of Arizona, Tucson, AZ, USA, \\ Email: robertof@email.arizona.edu \\ ${ }^{(7)}$ Centro de Biología Molecular, Universidad Autónoma de Madrid, Madrid, Spain, \\ Email: agfairen@cbm.uam.es \\ ${ }^{(8)}$ Department of Geosystem Engineering, University of Tokyo, Tokyo, Japan, \\ Email: miyamoto@geosys.t.u-tokyo.ac.jp \\ ${ }^{(9)}$ International Research School of Planetary Sciences, Università d'Annunzio, Pescara, Italy, \\ Email: goro@irsps.unich.it \\ ${ }^{(10)}$ Geomorphology and Pedology Laboratory, York University, Canada, \\ Email: arkose@rogers.com
}

Abstract - A fundamentally new (scientific) reconnaissance mission concept, termed tier-scalable reconnaissance, for remote planetary (including Earth) atmospheric, surface and subsurface exploration recently has been devised [1-5] that soon will replace the engineering and safety constrained mission designs of the past, allowing for optimal acquisition of geologic, paleohydrologic, paleoclimatic, and possible astrobiologic information of Venus, Mars, Europa, Ganymede, Titan, Enceladus, Triton, and other extraterrestrial targets $[6,7]$. This paradigm is equally applicable to potentially hazardous or inaccessible operational areas on Earth such as those related to military or terrorist activities, or areas that have been exposed to biochemical agents, radiation, or natural disasters. Traditional missions have performed local, ground-level reconnaissance through rovers and immobile landers, or global mapping performed by an orbiter. The former is safety and engineering constrained, affording limited detailed reconnaissance of a single site at the expense of a regional understanding, while the latter returns immense datasets, often overlooking detailed information of local and regional significance. $^{12}$

\section{TABLE OF CONTENTS}

\footnotetext{
1

${ }^{1} 1-4244-0525-4 / 07 / \$ 20.00$ @2007 IEEE.

${ }^{2}$ IEEEAC paper \#1199, Version 10, Updated December 8, 2006
}

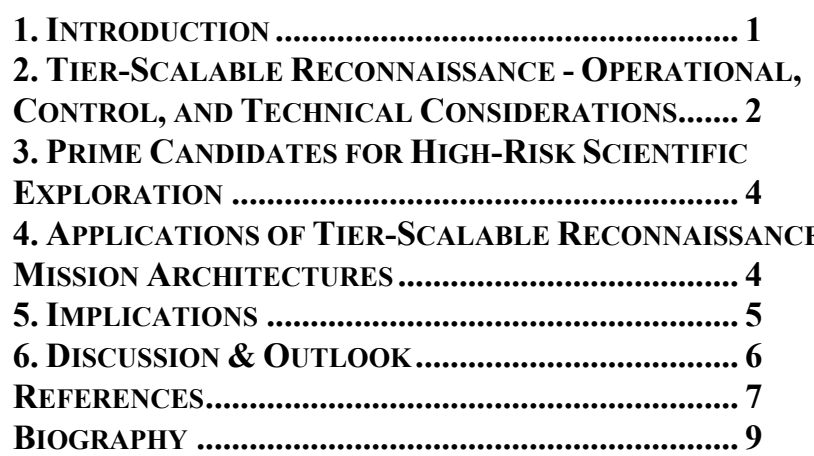

\section{INTRODUCTION}

Robotic reconnaissance operations are called for in extreme environments such as space, including planetary atmospheres, surfaces, and subsurfaces, as well as in potentially hazardous or inaccessible operational areas on Earth such as those related to military or terrorist activities, or areas that have been exposed to bio-chemical agents, radiation, or natural disasters. The concepts driving conventional robotic planetary missions of exploration are entirely inadequate for the next generation of missions that will be necessary to pave the way for human exploration back to the Moon and to Mars. Conventional robotic planetary exploration scenarios favor single lander/rover missions, which have been driven by safety and engineering 
constraints, at the expense of mission redundancy and science return. Furthermore, rovers are mobility constrained, unable to explore multiple sites on a planetary surface that are potentially distant from each other (a crucial feature for a geologist), and are unlikely to explore potentially hazardous but scientifically interesting surface and subsurface terrains, which have great potential to yield significant geologic, geomorphologic, pedologic, paleohydrologic, climatic, and possible exobiologic information. On the other hand, orbiters traditionally return immense datasets, while often overlooking detailed information of local and regional significance. With the advent of modern orbiter missions, such as ESA Mars Express and NASA Mars Reconnaissance Orbiter (MRO), high resolution optical instruments are now employed, such as the High Resolution Stereo Camera (HRSC) on Mars Express, and the High Resolution Imaging Science Experiment (HiRISE) on MRO. However, complete planetary surface coverage with these instruments is neither intended nor feasible in a timely manner, and follow-up investigations of regions of interest at high resolution are dependent on the respective orbit-parameters, making monitoring of transient events difficult.

To enable access to geologic sites not currently feasible on Mars or elsewhere, a completely new technological approach must be implemented. This is due to the need for mission redundancy, mission safety, greatly increased science return, and comparative analysis of spatio-temporal data gathered in transit, for optimal acquisition of geologic, paleohydrologic, paleoclimatic, and possible astrobiologic information of Mars and other extraterrestrial targets. For example, in order to optimally explore the vast canyon system Valles Marineris, and the ancient mountain range Thaumasia highlands of Mars (both may contain environments once conducive to life, such as sites of hydrothermal activity), a radical shift from traditional mission concepts and designs is not only overdue, but indeed required.

Here, we report on a recently devised approach, termed tierscalable reconnaissance, of integrated multi-tier (orbit $\Leftrightarrow$ atmosphere $\Leftrightarrow$ surface/subsurface) and multi-agent (orbiter(s) $\Leftrightarrow$ blimps $\Leftrightarrow$ rovers, landers, drills, sensor grids) hierarchical mission architectures [1-5] (see also Fig. 1). This paradigm not only introduces mission redundancy and safety, but enables intelligent, unconstrained, and distributed science-driven planetary exploration, including comparative analysis of spatio-temporal data gathered in transit, allowing for increased science return and paving the way towards fully autonomous robotic missions [7]. This paradigm shift opens up the opportunity for mainstream scientific research, affecting a wide scientific target audience, most notably planetary geologists, hydrologists, and astrobiologists, in addition to mission architects and roboticists. Essentially, this mission concept and design will facilitate the various disciplines to unite in order to achieve optimal reconnaissance of Venus, Mars, Titan, and beyond.

\section{TIER-SCALABLE RECONNAISSANCE -}

\section{Operational, CONTROL, AND TEChNiCAL}

\section{CONSIDERATIONS}

A typical operations scenario for a tier-scalable reconnaissance system is illustrated schematically in Fig. 1. Multi-tiered, multi-agent hierarchical integrated mission architectures allow for varying degrees of independence from human intervention, and also permit manual override at any level. A human operator may communicate to the orbiter(s), as well as command the airborne units via the orbiters (and thereby command the ground-level reconnaissance agents via the airborne units). Or, a highly automated operation mode may be used, enabling autonomous reconnaissance missions as they are necessary, when the communication time lag prohibits meaningful teleoperation, or, e.g., on the rear side of the Moon.

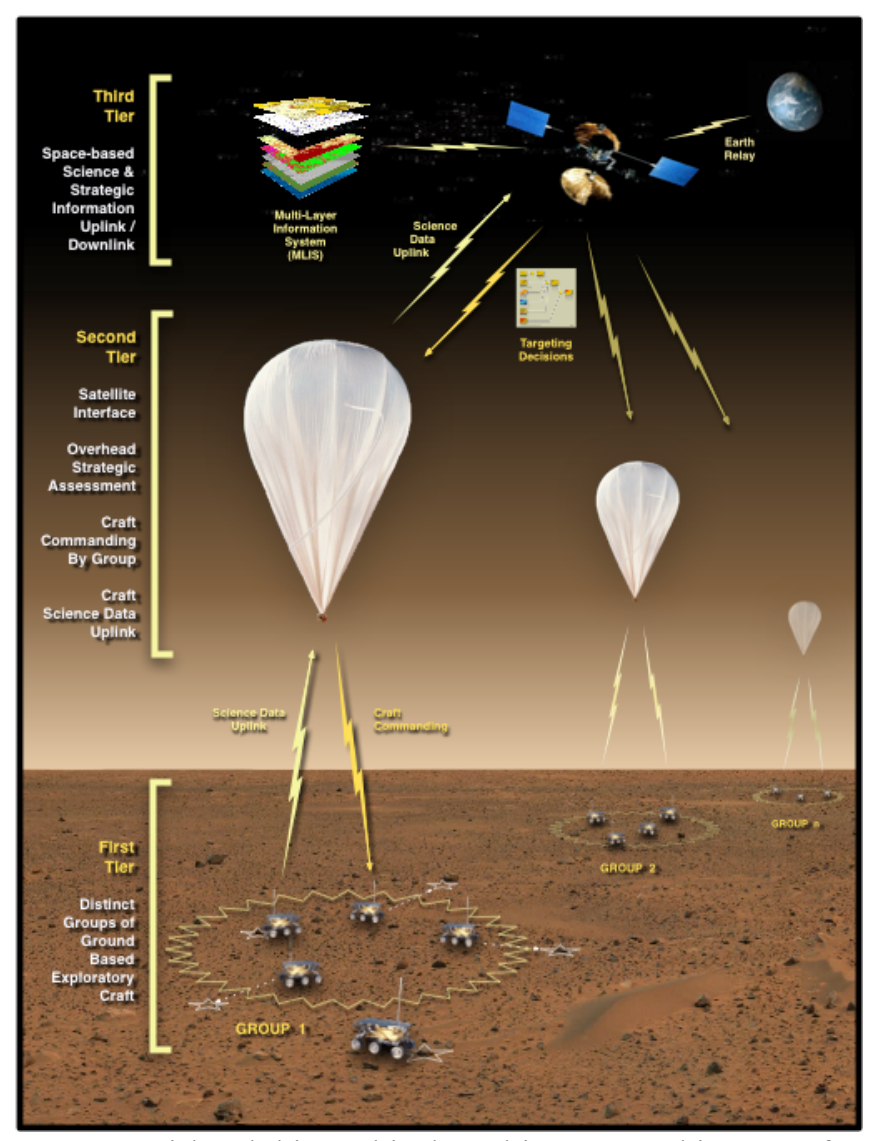

Fig. 1. Tri-level hierarchical multi-agent architecture for autonomous remote planetary exploration (from [1-5]).

The often overstated and misunderstood term autonomy is defined in the context of this paper as the high-level automation of planetary reconnaissance missions, including automated data acquisition, data feature extraction, data analysis, identification of science targets, science goal prioritization, execution of science goals, navigation, and 
guidance. As such, most currently deployed agents are not truly autonomous (with the exception for very basic and local obstacle avoidance), as they are for the most part Earth-commanded and teleoperated.

In the highly automated scenario, the satellites command and control the airborne agents autonomously, and the airborne agents autonomously command and control the ground-tier reconnaissance agents (Fig. 1). This system integrates satellites with balloons/blimps (airships) and ground-tier agents (rovers, fixed landers, e.g., Beagle 2, and sensors). The airborne and ground-tier agents can be inexpensive enough (in terms of capital cost and operational resources) to allow for the deployment of numerous, expendable agents (i.e., from the point of view of successfully achieving the mission objective(s)) that collectively can address specific science-driven questions. Examples of "inexpensive" agents are Micro-ElectroMechanical-Systems (MEMS)-based sensors, and minirovers akin to Minerva, the lander/mini-rover of the Japanese asteroid sample-return mission Hayabusa. Multiple ground-tier and airborne agents collectively can explore the same science target(s) with a complementary suite of instruments.

To support and control such tier-scalable reconnaissance mission architectures, a high degree of operation autonomy is required. Essential requirements of such operation autonomy are: (1) automatic mapping of an operational area from different vantage points (i.e., space, atmosphere, surface/subsurface); (2) automatic feature extraction and target/region-of-interest identification within the mapped operational area (e.g., Automated Geologic Field Analyzer (AGFA) [8]); and (3) automatic target prioritization for close-up examination (e.g., [8-12]) by, e.g., ground-tier agents, based on preliminary data, gathered in transit, potentially coupled with existing information from previous missions.

Multiple prioritization scenarios can be conceived to evaluate the (scientific) importance of individual targets or combinations of targets to be further examined during reconnaissance missions, which differ in their respective level of complexity. These scenarios can range from simple feature-based or feature-clustering-based prioritization (e.g., [13-15]) to prioritization via context-based clustering (e.g., $[16])$.

Previously gathered, coarse feature/reconnaissance data that have been pre-clustered using general purpose clustering algorithms (e.g., [13-15]) or clustering algorithms associated with special-purpose models (e.g., [16]), were recently used to devise more advanced prioritization frameworks (e.g., [9]). The latter facilitate (1) the selection of single or multiple targets, and (2) the selection of instruments used for the close-up examination of these targets in an operational area for potential information gain about the operational area.

In addition, the full-scale and optimal deployment of agents as part of a tier-scalable mission requires an intelligent reconnaissance system capable of integrating existing and acquired "in-transit" information to automatically perform smart planetary reconnaissance, such as homing in on prime candidate sites for potentially life-containing habitats on Mars [10-12]. To enable such a high level of on-board automation, a fuzzy-logic theoretical framework can be exploited [10-12] to design a fuzzy logic-based expert system capable of autonomously reasoning over multiple layers of information gathered while en-route and performing smart assessment of the observed areas to help decide the most appropriate hardware deployment (i.e., deployment of agents and sensors). Fuzzy logic is efficient in dealing with uncertainty and vagueness typical of real life scenarios and may represent a suitable platform to define the basic components of such an expert system. The geological approach, which compiles, synthesizes, and analyzes layers of diverse information (e.g., Multi-Layer Information System (MLIS) [1-3]) to identify prime targets for continued exploration [17, 18], is implemented as a set of IF-THEN rules representative of the desired expert knowledge [10-12]. For example, such rules can be effectively used by a fuzzy inference system to reason over water and/or life indicators to extract parameters such as "potential for water/life-containing", indicating the confidence exhibited by the system to find water and/or life at the observed locations.

If a planetary body has a sufficiently dense atmosphere, the deployment of an airborne tier is warranted for many reasons (see Section 4). In the past, airplanes/gliders (e.g., [19]) and even rotorcraft (e.g., [20, 21]) have been proposed. Some of the major deficiencies with these types of aerial vehicles are: (1) the complexity of the vehicles themselves (especially a rotorcraft); (2) the complexity of their deployment; (3) no station-keeping in the case of the airplane/glider and only temporary station-keeping in the case of a rotorcraft; (4) need for propellant (either electric by means of solar power or radioisotope thermoelectric generators (RTG), or chemical) for airplanes (except gliders) and rotorcraft; (5) (active) flight control system necessary; and (6) limited mission duration and termination of mission once vehicle lands/crashes on the surface (no repeated surface/subsurface probing possible, with the exception of the rotorcraft).

In contrast, balloons, blimps, or airships offer a lot of advantages over the above vehicle types. They are (1) simple in structure; (2) light-weight; (3) "easily" deployable (e.g., [22]), both in mid-air and from the surface; (4) buoyant without the need for propellant, (5) could operate without active flight control system (e.g., wind-driven), and (6) allow for extended mission durations, ranging from months to more than a year (e.g., [23]) and for repeated 
surface/subsurface sampling. Furthermore, equipped with either solar cells/batteries or RTGs, they can be electrically operated to support onboard instruments, data analysis capabilities, and active thrusting.

For airborne and potential airborne sample return missions using blimps/balloons, a basic estimation yields the following sample return masses and blimp/balloon sizes for Mars and Venus respectively (for details on the derivation of the lift-equation, the parameters used for the calculations, and for the assumptions made, see [7]):

- For a Mars net sample return mass of $100 \mathrm{~g}$ at an altitude of $0 \mathrm{~m}$, a blimp/balloon would need to be deployed with a radius $>8 \mathrm{~m}$ (this includes the He gas tank mass; without the tank this blimp/balloon would have a net liftable payload mass of about $15.8 \mathrm{~kg}$ ).

- For a Venus net sample return mass of $100 \mathrm{~g}$ at an altitude of $51 \mathrm{~km}$, a blimp/balloon would need to be deployed with a radius $>0.4 \mathrm{~m}$ (this includes the He gas tank mass; without the tank this blimp/balloon would have a net liftable payload mass of about $0.31 \mathrm{~kg}$ ).

The above results strongly suggest jettisoning the gas tank after deployment and inflation of a blimp/balloon in the martian atmosphere and to possibly maintain a smaller tank for altitude changes and gas replenishment for the duration of the mission. An exploration of Titan with airships (e.g., [23]) is an ideal scenario for a tier-scalable mission in conjunction with an orbiter and ground-probing agents, because of the 1.5 times thicker atmosphere compared to Earth and the colder temperatures compared to Venus' surface and near-surface atmospheric temperatures.

\section{Prime Candidates for High-Risk}

\section{SCIENTIFIC EXPLORATION}

Non-traditional autonomous missions to remote planetary bodies will be necessary $[1-5,7]$ primarily to allow intelligent and less constrained access to scientifically interesting targets on planetary bodies of the Solar System, not currently feasible with conventional mission designs, including: (1) canyons (e.g., Valles Marineris on Mars, or Devana Chasma, a big rift valley on Venus), (2) mountain ranges (e.g., Thaumasia highlands on Mars, Isthar Terra on Venus), (3) sites of suspected magmatic-driven uplift and associated tectonism and possible hydrothermal activity (e.g., plume-related activity such as hypothesized for the central part of Valles Marineris and the Warrego Valles rise on Mars [18, 24, 25], and Maxwell Montes on Venus), (4) polar ice caps (e.g., Mars), (5) suspected ice deposits within impact basins (e.g., Mercury and Moon) (e.g., [7]), (6) volcanoes of diverse sizes and shapes (e.g., Venus and Mars), (7) putative ancient accreted terrains and associated volcanism (e.g., Mars), (8) regions indicating potential recent hydrologic or hydrocarbon activities such as springfed seeps (e.g., Mars, Titan), (9) chaotic terrain (e.g., source areas of the circum-Chryse outflow channel system on Mars, Conamara Chaos on Europa), (10) liquid pools of ammonia-water mixtures associated with cryovolcanism or a recent impact cratering event (e.g., Titan, Triton, Enceladus), and (11) liquid hydrocarbon accumulation on the surface (e.g., Titan). These and many other targets of scientific interest on the planetary bodies of the Solar System, are particularly crucial for astrobiologic-oriented exploration in general, and sample return missions in particular $[6,7,26]$.

\section{ApPlications OF TIER-SCAlable}

\section{Reconnaissance Mission ArChitectures}

Integrated multi-tier, multi-agent hierarchical mission architectures are able to overcome the inherent challenge of traditional geologic planetary surface exploration [1-4]: airborne agents (orbiters in conjunction with balloons/blimps) possess overhead perspectives at different length scales/resolutions, which could provide guidance to ground-based agents (e.g., mobile rover units).

Tier-scalable reconnaissance missions not only introduce redundancy, and thus unprecedented mission reliability and safety, they also enable spanning larger surface areas than previously possible - mimicking the way geologists explore regions on Earth - and therefore allow for increased science return. Several example scenarios are outlined below [1]:

- Planetary bodies with atmospheres \& non-extreme surface temperatures (e.g., Earth and Mars):

Orbiter-guided deployment and control of balloon/blimp units, which in return deploy and control both mobile and immobile ground-based agents.

- Planetary bodies with atmospheres \& extreme surface temperatures (e.g., Venus and Titan):

Orbiter-guided deployment of balloon/blimp units. If surface temperatures permit, deployment and communication can be achieved with temperatureresistant immobile sensor webs or mobile agents.

- Planetary bodies w/o atmospheres \& extreme surface conditions (e.g., Mercury, Moon, Europa, Io):

Orbiter-guided deployment of and communication with ground-based mobile agents and immobile sensor webs.

In case of the central part of Valles Marineris, Melas Chasma (Fig. 2), the following deployment and reconnaissance sequence of such a reconnaissance mission is envisioned (see [1, 2, 7, 27-29] for further detail): Orbiter(s) with an embedded existing knowledge base (e.g., Multi-Layer Information System (MLIS) [1-3]) scout areas of scientific interest at a global to regional scale, i.e., within Valles Marineris and subsequently Melas Chasma. They subsequently deploy airborne agents (such as balloons, blimps, or airships, see also [22]) in mid-air above Melas Chasma for further tier-scalable reconnaissance, which 
includes identifying and homing in on prime targets of scientific interest and testing of hypothesized conditions, using both pre-mission and orbiter-based information. If one (or more) of the airborne agents were to detect scientifically interesting features while en-route, such as volatile releases (methane plume or water vapor) and/or elevated heat flow, or transient geologic events (e.g., a giant landslide that initiates on the walls of Valles Marineris), hydrologic events (e.g., water seeps), atmospheric events (e.g., reoccurring fog embankment in a specific part of the canyon system), and/or unique rock assemblages (other than the typically reported basaltic/basaltic-andesite, sulfates, and hematite, e.g., $[30,31])$, this airborne agent(s) would then home in on these features for close-up reconnaissance. It would attempt to map, characterize, and determine whether the features merit further evaluation through in-situ investigation by surface and/or subsurface agents (e.g., a plume of methane sourcing from a vent in a hydrothermally active area identified through tier-scalable reconnaissance based on diverse information, including concentration profiles of the atmosphere and AGFA/MLIS/Fuzzy Logicbased indicators of elevated heat flow, hydrothermal activity and associated deposits, surface and subsurface water, seismic activity, tectonic and fluvial structures, etc.).

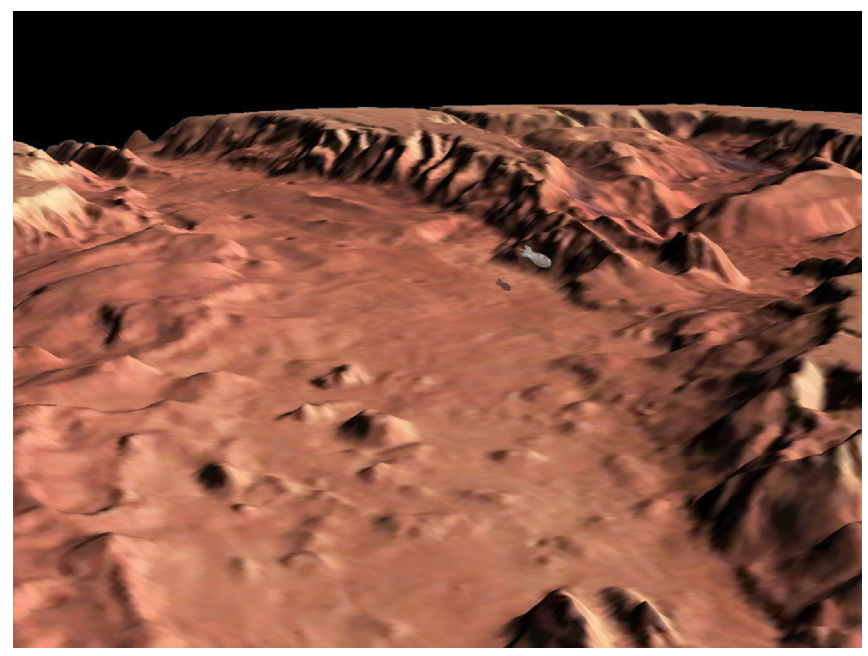

Fig. 2. 3-D oblique view, exemplifying an airborne agent (blimp/airship) performing intelligent reconnaissance over Melas Chasma (from [4]), the central part of the vast canyon system Valles Marineris on Mars. Part of the reconnaissance would include surveying the canyon walls, homing in on stratigraphic sequences, hovering above landslide and valley floor deposits, and identifying targets for subsequent deployment of ground-based agents such as miniature rovers and immobile sensors. Target features of special scientific interest may include: (1) geomorphic/pedologic features and mineralogical/elemental signatures, indicative of past water/weathering activities; (2) diversity of rock types (e.g., site on Mars containing rocks that record the early, middle, and later parts of martian history); (3) elevated heat flow; (4) surface/near-surface water or moisture (including fog embankments); and (5) volatile plumes (e.g., methane). These target features contribute to the success in identifying potential life-containing habitats. (Note that for visual purposes the blimp/airship is not drawn to scale).

The information acquired from the airborne vantage would subsequently be processed through automated featureextraction software packages (e.g., [8]). The feature data would be autonomously analyzed by science prioritization algorithms while en-route (e.g., [8-12]). This includes coupling existing information with the newly acquired information for comparative analysis (e.g., using a fuzzybased expert system), to choose potential targets for in-situ investigation and sampling by subsequently deployed ground-tier agents (small rovers, networks of sensors, etc.) and for determining safe passages to their respective designated targets within the prime sites, as identified from the airborne vantage. At the respective targets, the groundtier agents would conduct in-situ science experiments and thereby gather data that complement the remote sensing data obtained by the airborne agents. For example, the ground-tier agents would help identify, characterize, and map out sources of the volatile plumes (e.g., potential sites of extant hydrothermal activity). In addition, such a system could help direct ground-tier agents, potentially equipped with drills, to a locale of extant hydrothermal activity that records distinct elevated heat flow, mineral assemblages, near surface groundwater, volatile seepage such as water and methane vapors, etc., thereby paving the way for future sample return missions $[7,26]$.

\section{IMPLICATIONS}

Multi-tier, multi-agent autonomous robotic planetary atmosphere, surface and subsurface reconnaissance will lead to an improved understanding of the various histories (e.g., geologic, geomorphic, pedologic, aqueous, climatic, and possible biologic) of Mars and other extraterrestrial targets, through the tier-scalable geologic approach. Importantly, this new paradigm in planetary reconnaissance will integrate disciplines such as geology, biology, chemistry, physics, mathematics, and engineering, allowing for optimal reconnaissance and testing of overarching theories [32]. This includes confirming working hypotheses such as in the case of Mars, whether (a) the mountain ranges contain a greater diversity of rock types than just volcanic; (b) sites of suspected hydrothermal activity are indeed hydrothermal environments; (c) prime candidate sites of potential life-containing habitability actually contain extant or fossil life or life forms [17, 33, 34]; or (d) close examination of surface and subsurface rock materials with sensors suitable for microscopic observation and chemical analysis of coatings on weathered rock materials might reveal important data on possible soil microenvironments, live microbes, or fossil forms (e.g., [34, 35]). Moreover, tier-scalable autonomous reconnaissance missions afford a 
first-of-a-kind opportunity to scout, discover, and characterize potential habitats and possible life [7, 26].

Prioritization frameworks for single and multiple (science) targets, such as introduced in [9], may be useful for autonomously operating computer-based planning systems (e.g., onboard science craft such as satellite platforms, spacecraft, planetary orbiters, landers, rovers, etc.) to decide which previously detected and coarsely examined target or set of targets harbor the greatest potential for an overall information gain about an operational area if revisited or examined more closely. In addition, prioritization frameworks for (science) instrument usage, such as introduced in [9], may provide guidance as to which instrument out of a suite of available instruments onboard a science platform has the largest potential to contribute to the above information gain if used on these targets. Since instruments may differ in power consumption, data acquisition time, and spatial association between instrument and target, etc., a planning system can take into account these constraints together with the prioritization probabilities and may come up with optimized "target-toreexamine" and "instrument-to-use-for-reexamination" scenarios, thereby paving the way to more autonomous reconnaissance missions.

\section{Discussion \& OUTLOOK}

Following the published works by Fink et al. [1-3], NASA recently has been soliciting proposals calling for technology development for multi-tier sensor webs: "Sensor webs of the future may include space-based, airborne, and in-situ sensors, all working together in a semi-closed loop system in which "smart" sensors sense what is happening per their designed sensing capabilities and feed that information into a control system. Based on the sensor inputs, the control system then modifies the environment (instrument pointing, data collection on or off, etc.) and causes the sensors to take in and provide new information to the control system." (excerpt from Science Mission Directorate NASA Research Announcement "Advanced Information Systems Technology" Solicitation: NNH05ZDA001N-AIST). Moreover, in testimony to Congress in May 2005, NASA Administrator Michael Griffin included the following statement: "In the future, NASA plans to develop a "sensor web" to provide timely, on-demand data and analysis to users who can enable practical benefits for scientific research, national policymaking, economic growth, natural hazard mitigation, and the exploration of other planets in this solar system and beyond." This followed the release of the February 2005 publication The New Age of Exploration: NASA's Direction for 2005 and Beyond that stated: "NASA will develop new space-based technology to monitor the major interactions of the land, oceans, atmosphere, ice, and life that comprise the Earth system. In the years ahead, NASA's fleet will evolve into human made constellations of smart satellites that can be reconfigured based on the changing needs of science and technology. From there, researchers envision an intelligent and integrated observation network comprised of sensors deployed to vantage points from the Earth's subsurface to deep space."

Moreover, NASA's Mars Exploration Program in Washington has now fully embraced the tier-scalable reconnaissance concept first set forth by Fink et al. [1-3], as stated in a JPL News Release from October 06, 2006 [36]: "This is a tremendous example of how our Mars missions in orbit and on the surface are designed to reinforce each other and expand our ability to explore and discover, "said Doug McCuistion, director of NASA's Mars Exploration Program in Washington. "You can only achieve this compelling level of exploration capability with the sustained exploration approach we are conducting at Mars through integrated orbiters and landers." And Steve Squyres, the Principal Investigator for the two Mars Exploration Rovers Opportunity and Spirit, adds in the same JPL News Release [36]: "The combination of the ground-level and aerial view is much more powerful than either alone," said Steve Squyres of Cornell University, Ithaca, N.Y. [...] "If you were a geologist driving up to the edge of a crater in your jeep, the first thing you would do would be to pick up the aerial photo you brought with you and use it to understand what you're seeing from ground level. That's exactly what we're doing here."

Most recently, Steve Chien, who leads an automation effort ("Autonomous Sciencecraft Experiment") for Earth-orbiting satellites at JPL, now realizes in a NASA News Release from October 26, 2006 [37]: "We have four satellites orbiting Mars and two rovers on the ground. They could work together."

Individual components of the tier-scalable mission architecture proposed by Fink et al. [1-5, 26] are either under development or have already been tested and proven "in the field". These include orbiters, balloons/blimps/airships, and ground-based agents such as rovers and landers as well as immobile sensor webs. The biggest challenge, however, appears to be not so much the hardware but the "intelligent" software that would enable all the components of a multi-tier multi-agent mission to be integrated and function autonomously. This is not to be confused with Artificial Intelligence (AI)-based (i.e., rulebased) automation efforts, such as the Autonomous Sciencecraft Experiment (ASE) by Chien et al., that have to be told what to notice and how to then act accordingly, as explained by Steve Chien in the NASA News Release from October 26, 2006 [37]: "We programmed it to notice things that change [,,,] and take appropriate action."

Some of the authors of this contribution are developing, implementing, and field-testing software (e.g., [8-12]) that would allow the orbiters, blimps, and rovers both to communicate with one another and to navigate and explore 
the planetary terrain with greatly reduced (and ultimately without) help from mission control on Earth, thus affording more mission autonomy/flexibility and increased science return.

We envision that multi-tier multi-agent hierarchical mission architectures for remote planetary reconnaissance, as described here, are feasible within a 10-15 year timeframe. Efforts leading up to the implementation of tier-scalable reconnaissance missions will likely be international. Earthbound test beds for tier-scalable reconnaissance will become available within the next two years (being developed, implemented, and field-tested by some of the authors). A foretaste of what is to come is the anticipated interaction between MRO (and possibly Mars Express) with the Mars Exploration Rovers Spirit and Opportunity as well as with future lander and rover missions such as Phoenix and Mars Science Laboratory (MSL). Integrated orbiterairship missions, especially suitable for the exploration of Titan, Venus, and Mars, are envisioned to be feasible within a decade from now. Subsequent science-driven robotic exploration will couple this new paradigm in planetary reconnaissance with astronautic exploration and research.

\section{REFERENCES}

[1] Fink, W., et al., Next-Generation Robotic Planetary Reconnaissance Missions: A Paradigm Shift, Planetary and Space Science, 53, 1419-1426, 2005.

[2] Fink, W., et al., Next-Generation Robotic Planetary Surface/Subsurface Reconnaissance Missions: A Paradigm Shift [abstract 1977], in $36^{\text {th }}$ Lunar and Planetary Science Conference Abstracts [CD-ROM], Lunar and Planetary Institute, Houston, 2005.

[3] Fink, W., et al., Next-Generation Robotic Planetary Reconnaissance Missions: A Paradigm Shift, Geochimica et Cosmochimica Acta, Volume 69, Number 10S, A533, 2005 .

[4] Fink, W., et al., Autonomous Tier-Scalable Reconnaissance Missions For Remote Planetary Exploration, Proceedings of the $4^{\text {th }}$ International Planetary Probe Workshop 2006, Pasadena.

[5] Caltech's Visual and Autonomous Exploration Systems Research Laboratory Web site (by W. Fink) http://autonomy.caltech.edu/autonomy/tierscalable.html

[6] Schulze-Makuch, D. and Irwin, L.N. Life in the Universe: Expectations and Constraints, Springer, 2004.

[7] Schulze-Makuch, D., et al., Venus, Mars, and the Ices on Mercury and the Moon: Astrobiological Implications and Proposed Mission Designs, Astrobiology, 5, 778-795, 2005.

[8] Fink, W., et al., AGFA: (Airborne) Automated Geologic Field Analyzer, Geochimica et Cosmochimica Acta, Volume 69, Number 10S, A535, 2005.

[9] Fink, W., Generic Prioritization Framework for Target Selection and Instrument Usage for Reconnaissance Mission Autonomy, Proceedings of IEEE World Congress on Computational Intelligence (WCCI) 2006, Vancouver, Canada, 11116-11119.

[10] Furfaro, R., et al., Multi-Layer Fuzzy Logic-based Expert System for Conducting Tier-scalable Planetary Reconnaissance [abstract 1257], in $37^{\text {th }}$ Lunar and Planetary Science Conference Abstracts [CD-ROM], Lunar and Planetary Institute, Houston, 2006.

[11] Furfaro, R., et al., Fuzzy Logic Expert System for Tierscalable Planetary Reconnaissance, 9th International Conference on Space Operations, AIAA, Rome, Italy, June 19-23, 2006. 
[12] Furfaro, R., et al., Autonomy in Planetary Exploration: Fuzzy Expert System for Tier-Scalable Reconnaissance, abstract $25^{\text {th }}$ International Space Development Conference 2006, Los Angeles.

[13] Duda, R.O., et al., "Pattern Classification and Scene Analysis”, John Wiley \& Sons, $2^{\text {nd }}$ edition, 2000.

[14] Bishop, C.M., "Neural Networks for Pattern Recognition”, Clarendon Press, Oxford, 1995.

[15] Williams, C.K.I., "An MCMC Approach to Hierarchical Mixture Modelling", Advances in Neural Information Processing Systems 12, S. A. Solla, T. K. Leen, K.-R. Mueller, eds., MIT Press, 2000.

[16] Fink, W., et al., Clustering Algorithm for Mutually Constraining Heterogeneous Features, Technical Report JPL-ICTR-01-5, 2001.

[17] Dohm, J.M., et al., The Northwestern Slope Valleys (NSVs) region, Mars: A prime candidate site for the future exploration of Mars, Planetary Space Science, 52, 189-198, 2004.

[18] Dohm, J.M., et al., Geologic map of the Thaumasia region of Mars. US Geol. Survey Map I-2650, 2001.

[19] Colozza, A.J., Preliminary Design of a Long-Endurance Mars Aircraft, AIAA 90-2000, Proceedings of the AIAA/SAE/ASME/ASEE 26th Joint Propulsion Conference, July 16-18, 1990, Orlando, FL.

[20] Savu, G., Trifu, O., Photovoltaic rotorcraft for Mars missions, AIAA-1995-2644, ASME, SAE, and ASEE, Joint Propulsion Conference and Exhibit, 31st, San Diego, CA, July 10-12, 1995.

[21] Young, L.A., et al., Rotorcraft as Mars Scouts, Proceedings 2002 IEEE Aerospace Conference, Big Sky, MT, March 9-16, 2002.

[22] Nott, J., Ballutes: Launching Aerobots without Compromises, Proceedings of the $4^{\text {th }}$ International Planetary Probe Workshop 2006, Pasadena.

[23] Hall, J.L., et al., An aerobot for global in situ exploration of Titan, Advances in Space Research, Volume 37, Issue 11, 2108-2119, 2006.

[24] Dohm, J.M., et al., Ancient drainage basin of the Tharsis region, Mars: Potential source for outflow channel systems and putative oceans or paleolakes, J. Geophys. Res., 106, 32 943-32 958, 2001.
[25] Dohm, J.M., et al., Latent activity for western Tharsis, Mars: significant flood record exposed, J. Geophys. Res., $106,12,301-12,314,2001$.

[26] Schulze-Makuch, D., et al., Sample Return Missions to Mars, Venus, and the Ices on Mercury and the Moon [abstract 1324], in $37^{\text {th }}$ Lunar and Planetary Science Conference Abstracts [CD-ROM], Lunar and Planetary Institute, Houston, 2006.

[27] Fink, W., et al., Multi-tier Multi-agent Autonomous Robotic Planetary Surface/Subsurface Reconnaissance For Life [abstract 1433], in $37^{\text {th }}$ Lunar and Planetary Science Conference Abstracts [CD-ROM], Lunar and Planetary Institute, Houston, 2006.

[28] Dohm, J.M., et al., Tier-scalable Reconnaissance To Test Overarching Geological Theories and Locate Prime Targets on Mars, abstract $25^{\text {th }}$ International Space Development Conference 2006, Los Angeles.

[29] Fink, W., et al., Tier-Scalable Reconnaissance for Remote Planetary Exploration, abstract $25^{\text {th }}$ International Space Development Conference 2006, Los Angeles.

[30] Christensen, P.R., et al., Morphology and composition of the surface of Mars: Mars Odyssey THEMIS results, Science, 300, 2056-2061, 2003.

[31] Gendrin, A., et al., Sulfates in Martian Layered Terrains: The OMEGA/Mars Express View, Science, 307, Published online 17 February 2005; 10.1126/science.1109087, 2005.

[32] Baker, V.R., et al., A theory of early plate tectonics and subsequent long-term superplume activity on Mars, Superplume International Workshop, 312-316, 2002.

[33] Fairén, A.G., et al., Prime candidate sites for astrobiological exploration through the hydrogeological history of Mars, Planetary Space Science, 53, 1355-1375, 2005.

[34] Mahaney, W.C., et al., Ancient wet aeolian environments on Earth: clues to presence of fossil/live microorganisms on Mars, Icarus, 171: 39-53, 2004.

[35] Mahaney, W.C., et al., Morphogenesis of Antarctic paleosols: martian analogue, Icarus, 154: 113-130, 2001.

[36] Jet Propulsion Laboratory News Release from October 06, 2006: "NASA's Mars Rover and Orbiter Team Examines Victoria Crater" Web site: http://www.jpl.nasa.gov/news/news.cfm?release=2006121 
[37] Science@NASA News Release from October 26, 2006: "A Growing Intelligence Around Earth" Web site: http://science.nasa.gov/headlines/y2006/26oct_sensorweb. $\mathrm{htm}$

\section{BIOGRAPHY}

Wolfgang Fink is a Senior Researcher at NASA's Jet

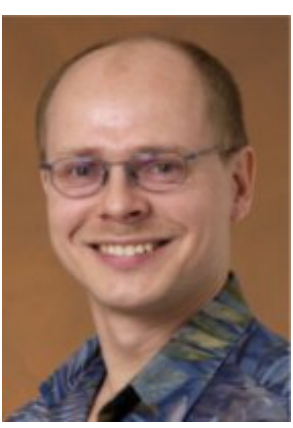
Propulsion Laboratory in Pasadena, CA, Research Associate Professor of both Ophthalmology and Neurological Surgery at the University of Southern California, Los Angeles, CA, and Visiting Associate in Physics at the California Institute of Technology, Pasadena, CA. He is the founder and head of the Visual and Autonomous Exploration Systems Research Laboratory at Caltech (http://autonomy.caltech.edu). His research interests include autonomous planetary and space exploration, computational field geology, image processing and analysis, sensor data fusion, astrobiology, and biomedicine. Dr. Fink obtained a B.S. and M.S. degree in Physics and Physical Chemistry from the University of Göttingen and a Ph.D. in Theoretical Physics from the University of Tübingen in 1997. His work is documented in numerous publications and patents. Dr. Fink is the recipient of the 2002 NASA Spaceflight Awareness Launch Honoree Award in support of human spaceflight, and the co-recipient of the Humie Silver Award 2005, awarded to the Evolutionary Computation Group at JPL, for demonstrating Human Competitive Performance for "Evolutionary Computational Techniques for the Automated Design of Space Systems" from the Genetic and Evolutionary Computation Conference (GECCO). In 2006 Dr. Fink won the Congress on Evolutionary Computation (CEC) 2006 "Huygens Probe Competition", held at the World Congress on Computational Intelligence (WCCI) 2006 in Vancouver, BC, Canada. Dr. Fink holds a Commercial Pilot's License for rotorcraft.

James M. Dohm is a Planetary Geologist and Senior

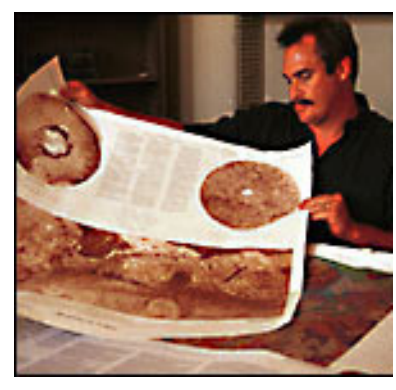
Researcher at the University of Arizona. He performs planetary investigations at local to global scales. He has extensive geological field experience coupled with more than 19 years of experience with planetary geological research, which includes 12 years as assistant coordinator of the NASA Mars and Venus Programs (MGM and VGM, respectively), now known as the Planetary Mapping Program. In addition, James Dohm has been recently involved with satellite and rover missions as a science team member of the Life in the Atacama Rover Field Experiment, Sensor Web, the "Subsurface Access" project of the Mars Technology Program, the Autonomous Sciencecraft Experiment, and the Nomad Rover Experiment. He has contributed to the publication of 6 USGS I maps at four map scales, more than 60 peer-reviewed journal articles, and more than five book publications.

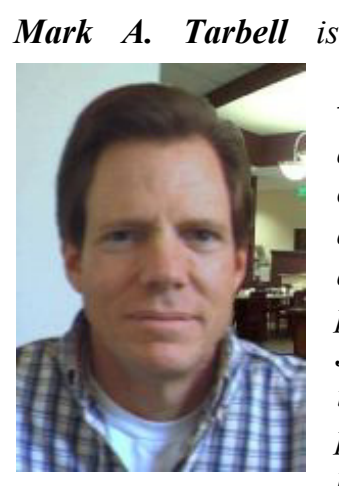

Specialist

with more than 15 years of satellite and ground-based command and control system architecture design and development. Tarbell designed and implemented the ground data processor control infrastructure for JPL's recent SRTM mission, and was involved with JPL'S Jason JTCCS project, which supports real-time telecommanding of Earth-orbiting satellites from wireless handheld PDAs. In collaboration with the Visual and Autonomous Exploration Systems Research Laboratory at Caltech, he recently co-designed and implemented a remote telecommanding control system for an indoor test bed for autonomous surface exploration at Caltech's Visual and Autonomous Exploration Systems Research Laboratory.

Trent M. Hare is an IT/GIS Specialist and has worked for

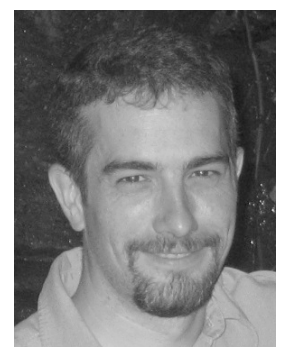
the Astrogeology Team at the United States Geological Survey in Flagstaff for 17 years. He has developed specialized Geographic Information Systems (GIS) applications and websites for several planetary bodies including Mars, The Moon, Venus, several Galilean Satellites, and Titan. And while his major tasks involve planetary projects, he has also been involved with many Earth-based projects including the creation of the USGS Global GIS digital data series and various country-wide geospatial projects.

Victor A. Baker is Regents' Professor of the University of

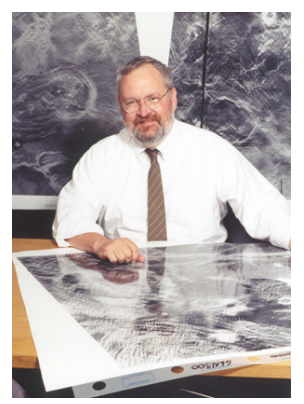
Arizona in the departments of hydrology and water resources, planetary sciences and geosciences. He has more than 30 years experience in planetary science research, particularly in geological studies of Mars and Venus. He also has had long experience with interpretive studies of terrestrial remote sensing, especially in regard to his specialties in fluvial geomorphology and flood hydrology. Dr. Baker is a Fellow of the American Geophysical Union, Honorary Fellow of the European 
Geosciences Union, Fellow of the American Association for the Advancement of Science, and Foreign Member of the Polish Academy of Sciences. He was the 1998 President of The Geological Society of America, and he holds the 2001 Distinguished Scientist Award from the Quaternary Geology and Geomorphology Division of that society. He is author or editor of 14 scholarly books or monographs, more than 300 scientific papers and chapters, and over 400 published abstracts and short reports.

Dirk Schulze-Makuch is Associate Professor at

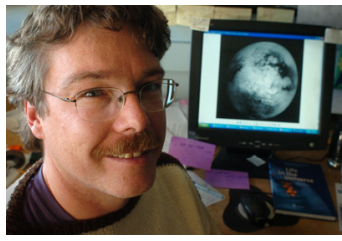
Washington State University. His most recent scientific accomplishments are the publication of his book "Life in the Universe: Expectations and Constraints" (Springer Publ., Berlin, 2004) along with many refereed papers in international journals. His research is centered on a broad range of topics with astrobiological relevance.

Roberto Furfaro is currently Assistant Research Professor

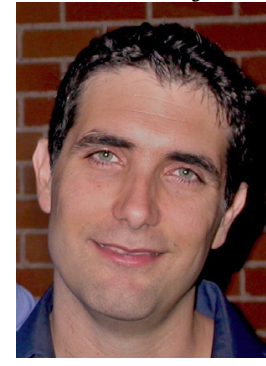
in the Aerospace and Mechanical Engineering Department, University of Arizona. He has a large spectrum of research interests, which includes neutron and photon computational transport, neural and fuzzy systems, space systems and micro-satellite design. Over the past few years, he has been collaborating with Ecosystem Science and Technology branch at NASA Ames on the "NASA Coffee Project" in which he led the development of an intelligent algorithm for coffee ripeness prediction using UAV airborne images. He has had a long-term involvement with Mars exploration since 1998 when he joined the NASA SERC at University of Arizona to become the project manager for the development of two robotic devices designed to utilize Martian local resources. Recently, he has been working developing of novel engineering solutions for planetary exploration including fuzzy-based expert systems for autonomous life-searching in extraterrestrial bodies.

Alberto G. Fairén obtained a Ph.D. in Molecular Biology

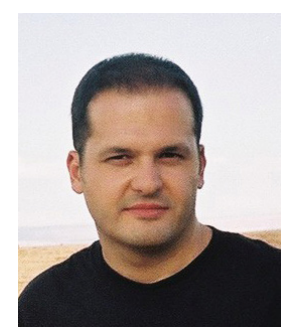
from the Universidad Autónoma de Madrid in Madrid, Spain. He is in the process of relocating to NASA Ames to start a postdoctoral scholarship with Chris McKay. His research investigations comprise Martian geochemistry, hydrogeological evolution, and astrobiology.
Ty P.A. Ferré is an Associate Professor in the Department

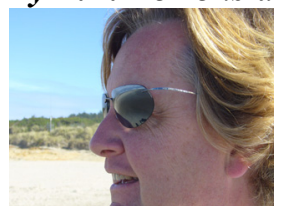
of Hydrology and Water Resources at the University of Arizona in Tucson. Ph.D., University of Waterloo, 1997. His interests are in identifying methods to improve the value of indirect measurements for hydrologic analysis. In particular, he is interested in probabilistic analyses that can make use of real and virtual data within a common analysis framework to assess the likely utility of measurements for hypothesis testing.

Hideaki Miyamoto is an Assistant Professor of the

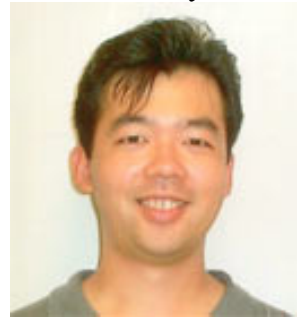
University of Tokyo, department of geosystem engineering and an affiliate scientist at Planetary Science Institute in Tucson, AZ. His research interests include surface processes and landforms on terrestrial planets in both geological and geophysical aspects. As a planetary geologist, he has been involved in most of Japanese solid-body exploration missions, including the Nozomi mission to Mars, the Hayabusa mission to an asteroid, and the SELENE mission to the Moon.

Goro Komatsu is a Research Professor at the International

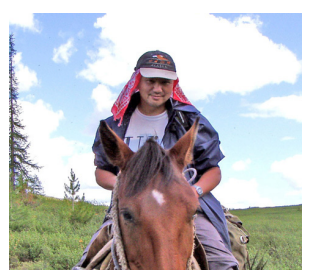
Research School of Planetary Sciences, an affiliate of Università d'Annunzio, Italy. He studies planetary surfaces (served on the US Magellan mission to Venus and the European Mars Express mission to Mars) and terrestrial geology of arid or cold climate zones (they are as remote as Mars).

William C. Mahaney is Professor of Geography at York

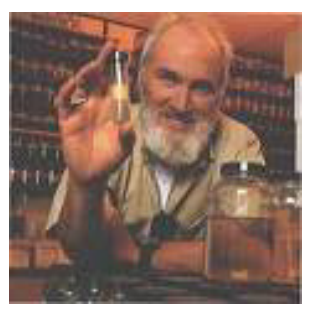
University in Toronto, Ontario, where he is Director of the Geomorphology and Pedology Laboratory. A specialist in glacial geology and geomorphology, he is the author of some 200-refereed papers and thirteen books, the most recent on the use of the Scanning Electron Microscope in sedimentology investigations published by Oxford University Press in 2002. He is also an environmental consultant and president of Quaternary Surveys, a company specializing in soil stratigraphy, engineering geology, Quaternary geology, terrain analysis and evaluation, environmental geomorphology and microstructural analysis by scanning electron microscope and energy-dispersive spectroscopy. Headquartered in Toronto, Professor Mahaney has undertaken investigations on every continent, his most recent research involving the geology/geomorphology of the invasion route from Spain to Italy during the Hannibalic Wars. 